\title{
Successful treatment of chylothorax and superior vena cava obstruction by radiotherapy
}

\author{
R W HEATON, I R ARNOLD, N HOWARD, A GUZ \\ From the Department of Medicine, Charing Cross and Westminster Medical School, and Department of \\ Radiotherapy, Charing Cross Hospital, London
}

Chylothorax, the accumulation of thoracic duct lymph in the pleural cavity, is an uncommon presentation of malignancy, but one that is generally regarded as indicating a grave prognosis. ${ }^{1}$ Chylothorax following obstruction of the superior vena cava, first described experimentally in dogs by Blalock et $a l^{2}{ }^{2}$ is a recognised, and frequently fatal, complication of central venous catheterisation. ${ }^{3}$ We describe a case of anaplastic carcinoma presenting with superior vena caval obstruction and chylothorax with a two year remission of both conditions after mediastinal irradiation.

\section{Case report}

The patient was a 70 year old woman who first presented in October 1982 with a one month history of rapidly increasing breathlessness, a non-productive cough, and weight loss, in association with retrosternal and right sided non-pleuritic chest pain. There was a 20 pack year smoking history. In 1975 she had undergone a right hemicolectomy for a Dukes stage B adenocarcinoma of the transverse colon. Examination showed a large, right sided pleural effusion and signs of superior vena cava obstruction. Pleural aspiration revealed milky fluid, and examination of the fluid removed after a $77 \mathrm{~g}$ fat meal showed increased fat globules and chylomicrons, confirming a chylous effusion secondary to leakage of thoracic duct lymph. ${ }^{4}$ Pleural biopsies were negative. $\mathbf{A}$ lymphangiogram (figure) showed dilated lymph vessels on the right side of the superior mediastinum but no leak to the right pleural space was seen. A superior vena cavogram showed a stenosis of the lower end of the right innominate vein and a tight obstruction of the lower superior vena cava, suggesting malignant infiltration. At mediastinotomy dense infiltration of the mediastinum was found. Biopsy of this area showed that anaplastic carcinoma had replaced lymph nodes. A barium enema and a liver scan showed no evidence of recurrence of the colonic carcinoma. The serum concentration of carcinoembryonic antigen was normal. She was treated with repeated pleurocenteses over a six week period and received a low fat diet with medium chain triglyceride supplements. ${ }^{5}$ She also had a course of radiotherapy to the mediastinum ( $4000 \mathrm{cGy}$ over 27 days). She showed considerable improvement, with resolution of the superior vena

Address for reprint requests: Dr RW Heaton, Department of Medicine, Charing Cross and Westminster Medical School, Fulham Palace Road, London W68RF.

Accepted 30 June 1986 cava obstruction and clearing of the pleural effusion and with no recurrence. Thereafter she remained well on a normal diet. She gained weight and was able to resume her normal life. A chest radiograph in February 1985 showed postirradiation changes only. One week later, however, she was readmitted with a large, left sided pleural effusion. Streptococcus pneumoniae was cultured from the sputum, blood, and pleural fluid. Despite treatment with antibiotics, rib resection, and drainage of the empyema she died three weeks after admission to hospital.

Postmortem examination showed extensive fibroelastic scarring in both lungs, particularly at the right apex.

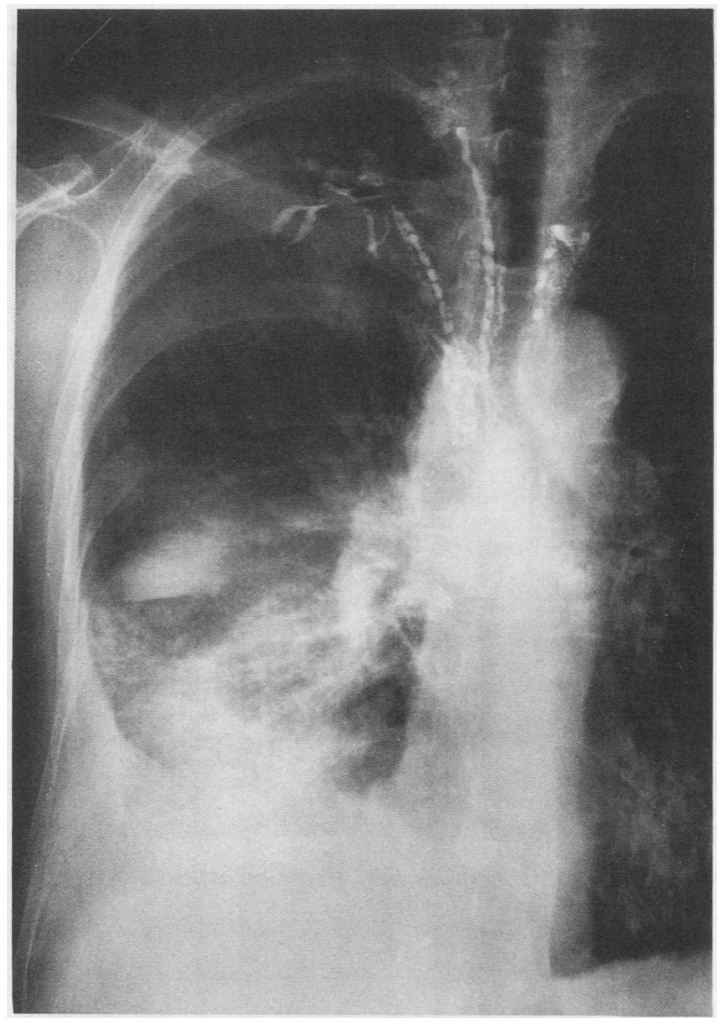

Lymphangiogram film taken four hours after injection, showing dilated lymph vessels in the mediastinum. 
Undifferentiated carcinoma was found in the subpleural region of the left lung and in a paratracheal lymph node. Histologically this resembled the tumour found by mediastinal biopsy. The mediastinum itself was free of tumour and there was no evidence of colonic carcinoma.

\section{Discussion}

Despite the high incidence of malignant cells within the thoracic duct lymph, chylothorax is an uncommon manifestation of malignant disease, even when there has been infiltration of the duct wall by malignant tissue. ${ }^{6}$ Lymphoma or lymphosarcoma produces the condition more commonly than carcinoma. ${ }^{17}$ When chylothorax does occur it is usually associated with a grave prognosis, with widespread malignancy, a requirement for repeated pleurocenteses, progressive wasting, and death. Bower, ${ }^{8}$ for example, described 20 cases of chylothorax, of which eight were secondary to malignancy. Seven of these patients rapidly became cachetic and died; the survivor was a patient with Hodgkin's disease who responded to chemotherapy. Robinson ${ }^{7}$ also described the poor outcome of patients with malignant disease and chylothorax despite appropriate antitumour treatment, and both he and Milsom et $a l^{9}$ advocated early surgical intervention, with thoracotomy and duct repair, in all cases of chylothorax, regardless of aetiology.

In our case chylothorax coincided with superior vena caval obstruction. Kausel et $\mathrm{al}^{6}$ described a case in which superior vena cava obstruction and chylothorax resulted from malignant obstruction, but reported cases of superior vena cava obstruction producing chylothorax have more commonly been secondary to surgical trauma or central venous catheterisation. In patients not undergoing thoracotomy and repair this is almost universally fatal. ${ }^{39}$ After the diagnosis of chylothorax in our patient conservative treatment failed to control the leakage while further investigations into the aetiology of the obstruction were undertaken. Radiotherapy to the mediastinum, however, resulted in resolution of the superior vena cava obstruction and of the chylothorax, with no evidence of recurrence over the succeeding two years. Relief of the superior vena cava obstruction alone is $\overline{\bar{C}}$ insufficient as a means of treating the chylothorax, as an actual perforation of the thorax duct must be present to® allow the lymph to leak. ${ }^{1}$ Irradiation of the tumour seems ton have allowed sealing of the duct to occur. This case shows that chylothorax and superior vena cava obstruction second-. ary to carcinoma can be successfully treated by non- $\overrightarrow{-}$ operative measures, and that these high risk patients do not $\omega$ have to be subjected to immediate thoracotomy to achieve a prolonged and satisfactory survival.

\section{References}

1 Bessone LN, Ferguson TB, Burford TH. Chylothorax. Ann Thorac Surg 1971;12:527-59.

2 Blalock A, Cunningham RS, Robinson CS. Experimental production of chylothorax by occlusion of the superior vena cava. Ann Surg 1936;104:359-64.

3 Seibert JJ, Golladay ES, Keller C. Chylothorax secondary to superior vena caval obstruction. Pediatr Radiol 1982;12:252-4. م

4 Seriff NS, Cohen ML, Samuel P, Schulster PL. Chylothorax: diagnosis by lipoprotein electrophoresis of serum and pleural $\overrightarrow{0}$ fluid. Thorax 1977;32:98-100.

5 Haskin SA, Roholt HB, Babayan VK, van Itallie TB. Treatment of chyluria and chylothorax with medium chain triglyceride. $N$ Engl J Med 1964;270:756-61.

6 Kausel HW, Reeve TS, Stein AA, Alley RD, Stranahan A. Anatomic and pathological studies of the thoracic duct. $J \overline{\bar{O}}$ Thorac Surg 1957;34:631-41.

7 Robinson CLN. The management of chylothorax. Ann Thorac $\frac{\mathrm{O}}{\mathrm{Q}}$ Surg 1985;39:90-5.

8 Bower GC. Chylothorax: observations in twenty cases. Dis Chest $\overline{\bar{\rho}}$ 1964;46:464-8.

9 Milsom JW, Kron IL, Rheuban KS, Rodgers BM. Chylothorax: an assessment of current surgical management. $J$ Thorac Cardiovasc Surg 1985;89:221-7. 